\title{
Measurement of Colonic Transit Time Based on Radio Opaque Markers in Patients with Chronic Idiopathic Constipation; A Cross-Sectional Study
}

\author{
Hazhir Saberi ${ }^{1}$, Nastaran Asefi ${ }^{1, *}$, Amir Keshvari ${ }^{2}$, Shahram Agah ${ }^{3}$, Mohsen Arabi ${ }^{4}$, Hoda \\ Asefi $^{1}$ \\ ${ }^{1}$ Department of Radiology, Tehran University of Medical Sciences, Tehran, IR Iran \\ ${ }_{3}^{2}$ Department of Surgery, Tehran University of Medical Sciences, Tehran, IR Iran \\ ${ }^{3}$ Department of Gastroenterology, Rasool Akram Hospital, Tehran, IR Iran \\ ${ }^{4}$ Department of Internal Medicine, Rasool Akram Hospital, Tehran, IR Iran \\ *Corresponding author: Nastaran Asefi, Department of Radiology, Tehran University of Medical Sciences, Tehran, IR Iran. Tel:+91-22688463, E-mail: nasefi76@gmail.com
}

Received: September 2, 2013; Revised: September 20, 2013; Accepted: September 25, 2013

\begin{abstract}
Background: Constipation is one of the most common gastrointestinal disorders particularly in industrialized countries. Incidence of constipation varies from $3.4 \%$ to $27.2 \%$ in different societies. Increase in urban population, industrialization of communities, changes in behavioral and nutritional habits and inactivity have increased the number of patients suffering from constipation.

Objectives: The aim of the study was to measure colonic transit time in patients with chronic idiopathic constipation.

Patients and Methods: Fifty-two patients with chronic idiopathic constipation (according to ROME III criteria) were selected. Patients with diabetes mellitus, hypothyroidism, and hypoparathyroidism were excluded. Each patient took a capsule containing ten 1-3 mm long angiographic guide daily for 6 days. Abdominal x-ray was performed on the seventh day. Remaining markers in each segment were counted and segmental and total colonic transit time was calculated. The analysis was performed by SPSS version 18 . In all tests, a P-value less than 0.05 was considered statistically significant.

Results: The mean age of patients was $36.9 \pm 10.1$ years. $80.8 \%$ of patients were female. The mean total colonic transit time was $40.8 \pm 35.1$ hours. $34.6 \%$ of patients and $36.5 \%$ of them had prolonged total and segmental colonic transit time.

Conclusions: We found that prolonged total and segmental colonic transit time is common in patients with chronic idiopathic constipation.
\end{abstract}

Keywords: Constipation; Habits; Hypothyroidism

\section{Background}

Constipation is one of the most common gastrointestinal disorders particularly in industrial countries $(1,2)$. Incidence of constipation varies from $3.4 \%$ to $27.2 \%$ in different societies (3). Increase in urban population, industrialization of communities, changes in behavioral and nutritional habits, and inactivity have increased the number of patients suffering from constipation (4). Incidence of constipation increases by aging, especially after 65 years of age $(3,5)$. In this age group $26 \%$ of males and $34 \%$ of females have constipation (6-8). The general incidence of constipation is twice in women. It is also more common in urban populations, lower socioeconomic classes, and in people with lower level of education $(3,9)$. Constipation has different definitions. It can be described as defecation less than three times a week; however, this definition is not widely accepted (10). International func- tional committee has suggested ROME III diagnostic criteria for the diagnosis of functional constipation $(11,12)$. Constipation has different etiologies and it can be the first clinical sign of metabolic disorders such as diabetes mellitus, hypoparathyroisism, hypercalcemia, neurologic disorders, obstructive bowel disorders and sometimes medications side effects $(13,14)$. Constipation would not be categorized as functional unless no specific etiology is determined. Chronic idiopathic constipation is usually seen in women. Symptoms would not be relieved by adding more fiber to the diet $(15,16)$.

Idiopathic constipation is divided into three subtypes: 1) Constipation with normal colonic transit, which is often associates with psychosocial abnormalities (17, 18);2) Colonic inertia (slow transit constipation). Most of the patients with severe constipation and abnormal colonic transit have colonic inertia, that in this case, transit of particles via colon is slow; 3 ) Outlet obstruction, in which

Implication for health policy/practice/research/medical education:

The aim of the present study was to measure colonic transit time in patients with chronic idiopathic constipation.

Copyright @ 2013, Iranian Red Crescent Medical Journal; Published by Kowsar Corp. This is an open-access article distributed under the terms of the Creative Commons Attribution License, which permits unrestricted use, distribution, and reproduction in any medium, provided the original work is properly cited. 
food particles pass normally through the colon but slowdown in rectum $(19,20)$. The first step in the evaluation of constipation is obtaining a precise history of underlying medical disorders such as diabetes mellitus, hypoparathyroidism, hypothyroidism, and hypercalcemia and if needed, measuring the serum levels of TSH, T4, AlkP, P, Ca, FBS, and CBC and stool exam.

Other diagnostic evaluations include colonoscopy, barium enema, and evaluation of colonic transit time (CTT) by marker and anorectal manometer (21-23). Evaluation of CTT is a useful method for chronic idiopathic constipation. In this method, the patient takes a specific number of radio opaque markers and abdominal X-ray is performed at the determined time $(24,25)$. Performing CTT makes is possible to specify which colonic segment has abnormal motility and is the cause of constipation (26). It is also useful in distinguishing patients with Irritable bowel syndrome (IBS) that may benefit from subtotal colectomy and ileorectostomy $(27,28)$.

\section{Objectives}

We aimed to distinguish subtypes of chronic idiopathic constipation by evaluating the colonic transit time, so that the appropriate treatment would be assigned to each patient.

\section{Patients and Methods}

In this cross-sectional study, we evaluated patients with constipation that were referred to the gastrointestinal and surgical clinics of Imam Khomeini hospital complex, Tehran, Iran. Patients with the history of known diabetes mellitus, hyperparathyroidism, hypoparathyroidism, and hypothyroidism were excluded. Patients were included according to ROME III criteria (Table 1 )

Table 1. Diagnostic Criteria for Functional Constipation

\begin{tabular}{l} 
Presence of Defecation Discomfort During at Least 3 Months in the Past 6 Months \\
\hline 1) Two or more of the following symptoms \\
a) Straining during at least $25 \%$ of defecation \\
b) Lumpy or hard stools in at least $25 \%$ of defecation \\
c) Sensation of incomplete evacuation for at least $25 \%$ of defecation \\
d) Sensation of anorectal obstruction or blockage for at least $25 \%$ of defecation \\
e) Manual maneuvers to facilitate at least $25 \%$ of defecation (e.g. digital evacuation, support of pelvic floor) \\
f) Fewer than three episodes of defecation per week
\end{tabular}

2) Loose stools are rarely present without use of laxatives

3) Insufficient criteria for irritable bowel syndrome: (recurrent abdominal pain or discomfort at least 3 days/month in the last 3 months associated with two or more of the following

a) Improvement with defecation

b) Onset associated with a change in the frequency of defecation

c) Onset associated with a change in the stool appearance)

Although CTT could be performed in different ways, we chose the Arhan method because of single X-ray exposure and no need for repeated referal to the radiology department. In this method, the patients were given capsules containing ten 1-3 millimeter-long marker made of angiographic catheter. Patients should not have used laxatives during the week before CTT study. They took a capsule at 9 am for six consecutive days and came for taking abdominal X-ray in the 7th day morning.

All the plain abdominal X-rays were evaluated by a single radiologistand the number of markers in each segment (right, left and rectosigmoid) was counted as shown in Figure 1.

Total, right, left and rectosigmoid colonic transit time was computed using this formula:

$\mathrm{CTT}=2.4 \times \mathrm{N}$

$\mathrm{N}=$ number of available markers in each segment or totally

The data was then analyzed using the statistical soft- ware SPSS 18.0.0. (SPSS Inc. Chicago, IL, USA). The t test was used to compare two groups and P-value lower than 0.05 was considered significant.

\section{Results}

Fifty-two patients fulfilled the inclusion criteria. The mean age of patients was $36.9 \pm 10.1$ years. (Range: $18-66$ years). Forty-two patients (80.8\%) were female. We also found that 28 patients (53.8\%) had history of chronic constipation for more than 3 years. In this study, the mean total colonic transit time was $40.8 \pm 35.1$ hours while in rectosigmoid, ascending colon and descending colon, it was $15.5 \pm 12.4,14.7 \pm 10.8$ and $12.9 \pm 9.5$ hours, respectively (Table 1). In 18 patients (34.6\%), total colonic transit time was longer than normal ( $\geq 45$ hours) (Table 2 ).

From 34 patients with normal total colonic transit time (13), 19 patients (36.5\%) had abnormal segmental transit time ( $\geq 15$ hours). among these patients, 10 had pro- 
longed right colonic transit time, while 6 and 3 patients had prolonged rectosigmoid and left colonic transit time, respectively.

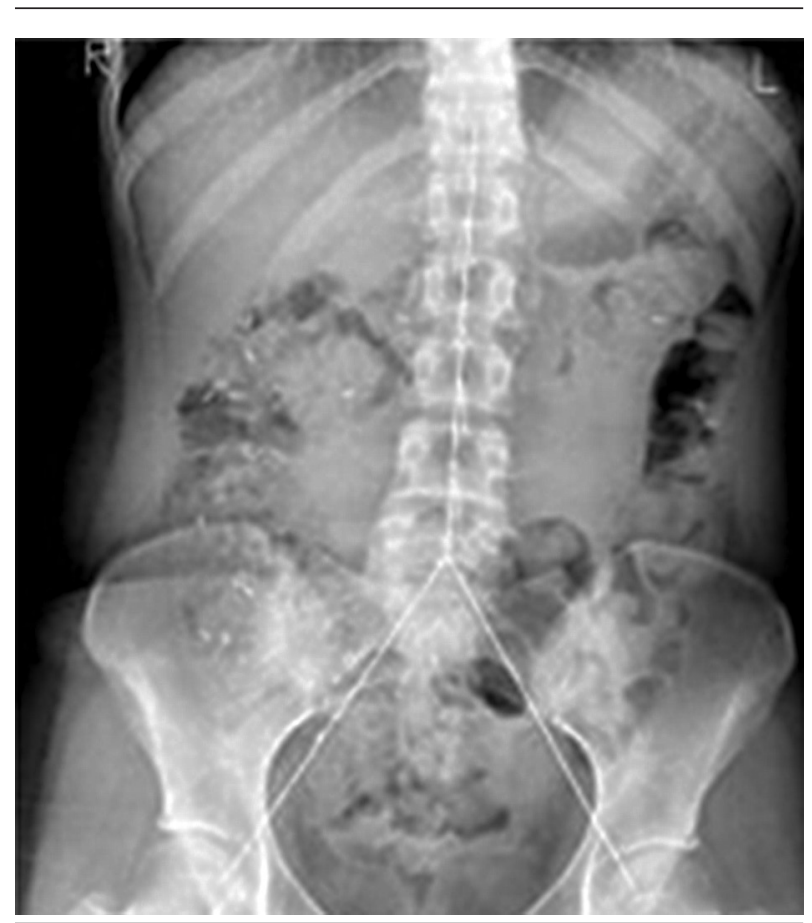

Figure 1. The plain Abdominal x-ray is Divided into 3 Segments and Radiopaque Markers are Counted in Each Segment

Table 2. Frequency of Normal and Abnormal Colonic Transit Time

\begin{tabular}{lll}
\hline $\begin{array}{l}\text { Total Colonic } \\
\text { Transit Time }\end{array}$ & Number & Percent \\
\hline Normal $(<\mathbf{4 5} \mathbf{~ h r})$ & 34 & 65.4 \\
$\begin{array}{l}\text { Abnormal }(>\mathbf{4 5} \\
\text { hr })\end{array}$ & 18 & 34.6 \\
Total & 52 & 100 \\
\hline
\end{tabular}

Table 3. Mean Colonic Transit Time in Different Age Groups

\begin{tabular}{cll}
\hline Age, $(\mathbf{y})$ & Number & Mean \pm SD $(\mathbf{h})$ \\
\hline$\leq \mathbf{3 0}$ & 12 & $38.6 \pm 36.2$ \\
$\mathbf{3 0 - 4 0}$ & 22 & $39.1 \pm 36.7$ \\
$\mathbf{4 0 - 5 0}$ & 13 & $44.2 \pm 40.4$ \\
$\mathbf{2 5 0}$ & 5 & $49.4 \pm 42.1$ \\
\hline
\end{tabular}

Regarding the mean total colonic transit time, no significant difference was found among different age groups and pair comparing $(\mathrm{P}>0.05)$. there was no statistically significant difference with regard to the mean total colonic transit time between patients with less or equal to three years history of constipation (38.6 \pm 17.08 hours) and those with more than three years history of constipation $(\mathrm{P}=0.67)$.

\section{Discussion}

To put in a nutshell, the aim of this study was to evaluate colonic transit time in patients with chronic idiopathic constipation.

In our study, among 52 patients who entered the study, $18(34.6 \%)$ had prolonged total colonic transit time. In similar studies, the frequency was different in patients with idiopathic constipation (29). In a study published by Lopes et al. (30) in 2008, of 30 patients with constipation, eight patients had prolonged total colonic transit time, which was similar to our study findings. in contrast, in a study by Mollen et al. (31) of 112 patients with constipation, 70\% had prolonged total colonic transit time, which was higher than the rate of this condition in our patients (34.6\%). In contrary to our study, they obtained the x-ray images after 10 days of administrating radio opaque markers, while we took the images on the seventh day. In our study, $36.5 \%$ of patients with normal total colonic transit time had prolonged segmental transit time. In 10 patients' right colon was affected while in six and three patients, rectosigmoid and left colon were affected, respectively.

Lopes et al. (30) found that nine, eight, and three out of 22 patients with normal total colonic transit time, had isolated slow transit in right colon, rectosigmoid, and left colon, respectively. The frequency of prolonged segmental colonic transit time was higher in right colon which was similar to our findings. The mean segmental colonic transit time was longer in rectosigmoid in our study. In a similar study by Zaslavsky et al. (32) on 13 patients with constipation, the mean total colonic transit time was $58.6 \pm 17.4$ hours while segmental colonic transit time in rectosigmoid, right, and left colon were 17.2 \pm 16.2 , $15.9 \pm 12.4$, and $14.7 \pm 13.4$, respectively.

With regard to longer mean transit time in right colon, subtotal surgery could be more helpful in the case of resistance to medical treatment. The mean total colonic transit time was higher in women in our study but this was not statistically significant $(P \geq 0.05)$. Danquechin et al. (33), measured colonic transit time in 82 healthy people, and found that total colonic transit time in women was longer than men.

In conclusion, abnormal total and segmental colonic transit time is common in patients with chronic idiopathic constipation; hence, in the case of inappropriate response to medical treatment, subtotal colectomy would be helpful.

\section{Acknowledgements}

There is no acknowledgment.

\section{Author's Contribution}

Authors have participated equally in the present study.

\section{Funding/Support}


The study is self-funded.

\section{Financial Disclosure}

There is no conflict of interest.

\section{References}

1. Preston D M, Lennard-Jones J E. Severe chronic constipation of young women: 'idiopathic slow transit constipation'. Gut. 1986;27(1):41-48.

2. Barnes PR, Lennard-Jones JE. Balloon expulsion from the rectum in constipation of different types. Gut.1985;26(10):1049-52.

3. Beck DE. Evaluation and management of constipation. Ochsner J. 2008;8(1):25-31.

4. Lux G, Orth KH, Bozkurt T, Stabenow-Lohbauer U. [Diagnostic strategy in constipation, including irritable bowel syndrome]. Ther Umsch. 1994;51(3):177-89.

5. Azizpour Y, Shohani M, Sayehmiri K, Kikhavani S. A Survey on the Associated Factors of Stress among Operating Room Personnel. Thrita. 2013;2(1):19-23.

6. Talley NJ, Fleming KC, Evans JM, O'Keefe EA, Weaver AL, Zinsmeister AR, et al. Constipation in an elderly community: a study of prevalence and potential risk factors. Am J Gastroenterol. 1996;91(1):19-25.

7. Talley NJ, O'Keefe EA, Zinsmeister AR, Melton LJ, 3rd. Prevalence of gastrointestinal symptoms in the elderly: a population-based study. Gastroenterology. 1992;102(3):895-901.

8. Chuang TJ, Chen CW, Lin HY, Hsu WH, Wang SC, Tu CC. Acute Appendicitis Presenting as Unusual Left Upper Quadrant Pain. Iran J Radiol. 2013;10(3):156-159.

9. Han NY, Park BJ, Sung DJ, Kim MJ, Cho SB, Kim DS, et al. Colloid Carcinoma of the Extrahepatic Biliary Tract with Metastatic Lymphadenopathy Mimicking Cystic Neoplasm: A Case Report. Iran J Radiol. 2013;10(2):90-3.

10. Sandler RS, Drossman DA. Bowel habits in young adults not seeking health care. Dig Dis Sci. 1987;32(8):841-5.

11. Longstreth GF, Thompson WG, Chey WD, Houghton LA, Mearin F, Spiller RC. Functional bowel disorders. Gastroenterology. 2006;130(5):1480-91.

12. Mohseni MG, Hosseini SR, Salavati A, Dadgari S. Ureterocele Associated with Renal Agenesia Presented as a Pelvic Mass in an Adult. Iran J Radiol. 2013;10(1):45-47.

13. Singh G, Lingala V, Wang H, Vadhavkar S, Kahler KH, Mithal A, et al. Use of health care resources and cost of care for adults with constipation. Clin Gastroenterol Hepatol. 2007;5(9):1053-8.

14. Stewart WF, Liberman JN, Sandler RS, Woods MS, Stemhagen A, Chee E, et al. Epidemiology of constipation (EPOC) study in the United States: relation of clinical subtypes to sociodemographic features. Am J Gastroenterol. 1999;94(12):3530-40.

15. Nyam DC, Pemberton JH, Ilstrup DM, Rath DM. Long-term results of surgery for chronic constipation. Dis Colon Rectum. 1997;40(3):273-9.

16. Mazioti A, Gatselis KN, Rountas C, Zachou K, Filippiadis KD, Tepetes K, et al. Safety and Efficacy of Transcatheter Arterial Chemoemboliazation in the Real-Life Management of Unresectable
Hepatocellular Carcinoma. Hepat Mon. 2013;13(8)

17. Wald A, Hinds JP, Caruana BJ. Psychological and physiological characteristics of patients with severe idiopathic constipation. Gastroenterology. 1989;97(4):932-7.

18. Yahyazadeh SH, Malekpour N, Mousavi M, Molanayi S, Salmanian B, Mohamadi A. Malignant Retroperitoneal Hemangiopericytoma: Report of a 73 Years Old Woman and Review of Literature. Thrita. 2013;2(2):80-3.

19. Martelli H, Devroede G, Arhan P, Duguay C. Mechanisms of idiopathic constipation: outlet obstruction. Gastroenterology. 1978;75(4):623-31.

20. Hafezi Ahmadi M, Teimouri H, Alizadeh S. The liver Metastatic Adenocarcinoma of Colorectal Cancer With Synchronous Isolated Hepatic Tuberculosis. Hepat Mon. 2013;13(5).

21. American Gastroenterological Association medical position statement on anorectal testing techniques. Gastroenterology. 1999;116(3):732-735.

22. Alehossein M, Babaheidarian P, Salamati P. Comparison of different modalities for reducing childhood intussusception. Iran $J$ Radiol. 2011;8(2):83-7.

23. Sabzi Sarvestani A, Zamiri M. Presacral Schwannoma Causing Infertility in a Young Woman: Report of a Case. Scimetr. 2013;1(1).

24. Pescatori M. Imaging Atlas of the Pelvic Floor and Anorectal Diseases: Springer; 2009.

25. Abbasinazari M, Behnava B, Panahi Y, Hajhossein Talasaz A, Salimi S, Keshvari M, et al. Plasma Zinc Level in Hepatitis C Patients With or Without Beta Thalassemia Major; Is There Any Difference? Hepat Mon. 2013;13(8).

26. Pomerri F, Frigo AC, Grigoletto F, Dodi G, Muzzio PC. Error count of radiopaque markers in colonic segmental transit time study. AJR Am J Roentgenol. 2007;189(2):W56-9.

27. Bouchoucha M, Devroede G, Dorval E, Faye A, Arhan P, Arsac M. Different segmental transit times in patients with irritable bowel syndrome and "normal" colonic transit time: is there a correlation with symptoms? Tech Coloproctol. 2006;10(4):287-96.

28. Motovali-Bashi M, Biglari M, Rezaei H, Dehghanian F. CYP1B1 L432V Polymorphism and Lung Cancer Risk in the Iranian Population. Iran J Biotech. 2013;11(3):199-4.

29. Moreno-Osset E, Ballester J, Minguez M, Mora F, Benages A. [Colonic transit time (segmental and total) in healthy subjects and patients with chronic idiopathic constipation]. Med Clin (Barc). 1992;98(6):201-6.

30. Lopes AC, Victoria CR. Fiber intake and colonic transit time in functional constipated patients. Arq Gastroenterol. 2008;45(1):5863.

31. Mollen RM, Claassen AT, Kuijpers JH. Measurement of colon transit time useful in the evaluation of functional constipation. Ned Tijdschr Geneeskd. 1998;142(7):357-61.

32. Zaslavsky C, da Silveira TR, Maguilnik I. Total and segmental colonic transit time with radio-opaque markers in adolescents with functional constipation. J Pediatr Gastroenterol Nutr 1998;27(2):138-42.

33. Danquechin Dorval E, Barbieux JP, Picon L, Alison D, Codjovi P, Rouleau P. Simplified measurement of colonic transit time by one radiography of the abdomen and a single type of marker. Normal values in 82 volunteers related to the sexe. Gastroenterol Clin Biol. 1994;18(2):141-4. 